\title{
Improvement of Reduced Order Modeling based on POD
}

\author{
M. Bergmann ${ }^{1}$, C.-H. Bruneau ${ }^{2}$, and A. Iollo ${ }^{3}$ \\ 1 INRIA Bordeaux Sud Ouest, Team MC2 and Université de Bordeaux, UMR \\ 5251, Bordeaux, F-33000, France michel.bergmann@inria.fr \\ 2 Same address charles-henri.bruneau@math.u-bordeaux1.fr \\ 3 Same address angelo.iollo@math.u-bordeaux1.fr
}

Summary. This study focuses on stabilizing reduced order model (ROM) based on proper orthogonal decomposition (POD) and on improving the POD functional subspace. A modified ROM that incorporates directly the pressure term is proposed. The ROM is stabilized using Navier-Stokes equations residuals and exploiting ideas similar to the variational multiscale method. The POD functional subspace is improved thanks to an hybrid method that couple DNS and POD ROM. Results are shown for a $2 \mathrm{D}$ confined cylinder wake flow.

\section{Introduction}

This paper focuses on improving reduced order modeling (ROM) based on proper orthogonal decomposition (POD). Besides the possible inherent lack of numerical stability of POD/Galerkin methods [10], the main shortcomings are the following. Firstly, since in most of the POD applications the ROM is built from a velocity database it is necessary to model the pressure term $[8,5]$. To overcome this difficulty, a pressure extended ROM is introduced in $\S 2$, so that the pressure term can be directly approximated using the pressure mode. Secondly, due to the energetic optimality of the POD basis, only few modes are sufficient to give a good representation of the flow kinetic energy. However, the viscous dissipation mainly takes place in the small unresolved eddies. A ROM built with very few modes is thus not able to dissipate enough energy. It is then necessary to close the ROM by modeling the effects of the unresolved modes. In this study, we use the residuals of the Navier-Stokes (NS) equations (§3) and exploit ideas similar to the variational multiscale method (VMS) [1]. Finally, since POD basis functions are optimal to represent the main characteristics of the flow configuration used to build them, the same basis functions are not optimal to represent the main characteristics of other flow configurations $[9,7,3]$. To overcome this problem, we propose an hybrid method that couples DNS and ROM to adapt the POD basis functions at low numerical costs $(\S 4)$. 


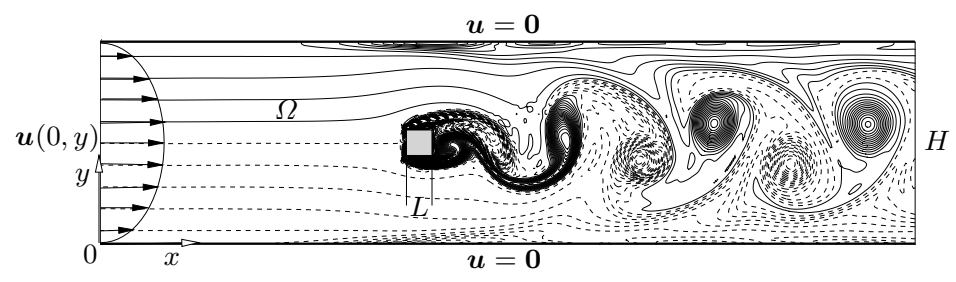

Fig. 1. Flow configuration and vorticity snapshot at $R e=200$.

Our paradigm is the confined square cylinder wake flow (figure 1) in laminar regime, i.e. at Reynolds number $R e=U_{\infty} L / \nu \leq 200$, with $U_{\infty}=$ $u(0, H / 2), L$ the lenght of the side of the square cylinder and $\nu$ the kinematic visosity. We use the same parameters as those introduced in [5].

\section{A pressure extended Reduced Order Model}

It has been proven [8] that neglecting the pressure term can lead to large ROM errors. One solution is to model this pressure term $[8,5]$. The pressure term can also be calculated using a pressure extended ROM with $p=\widetilde{p}$. Indeed, the POD flow fields write $\widetilde{\boldsymbol{u}}(\boldsymbol{x}, t)=\sum_{i=1}^{N_{r}} a_{i}(t) \phi_{i}(\boldsymbol{x})$ and $\widetilde{p}(\boldsymbol{x}, t)=$ $\sum_{i=1}^{N_{r}} a_{i}(t) \psi_{i}(\boldsymbol{x})$, see [2] for more details. Moreover, it is possible to evaluate the Navier-Stokes residuals $(\S 3)$. The ROM, noted $A^{\left[N_{r}\right]}$, is:

$$
\sum_{j=1}^{N_{r}} L_{i j} \frac{\mathrm{d} a_{j}}{\mathrm{~d} t}=\sum_{j=1}^{N_{r}} B_{i j} a_{j}+\sum_{j=1}^{N_{r}} \sum_{k=1}^{N_{r}}, C_{i j k} a_{j} a_{k}
$$

where the ROM coefficients are given in [2]. The $A^{\left[N_{r}\right]}$ model is tested at $R e=200$. The POD snapshot method introduced by Sirovich [11] was used. Here, only the first 5 modes are sufficient to represent more than $98 \%$ of the kinetic energy. As it is shown in figure 2, the solution of model (1) built with 5 modes reaches erroneous limit cycles, and can even diverge with 3 modes.

\section{Stabilization of reduced order models}

The aim of this section is to derive stabilization methods that involve very few empirical parameters. The two kinds of stabilization methods presented in what follows use the residual of the Navier-Stokes operator evaluated with the POD flow fields $\widetilde{\boldsymbol{u}}$ and $\widetilde{p}$. These residuals, called POD-NS residuals, are:

$$
\begin{aligned}
& \boldsymbol{R}_{M}(\boldsymbol{x}, t)=\frac{\partial \widetilde{\boldsymbol{u}}}{\partial t}+(\widetilde{\boldsymbol{u}} \cdot \nabla) \widetilde{\boldsymbol{u}}+\nabla \widetilde{p}-\frac{1}{R e} \Delta \widetilde{\boldsymbol{u}} \\
& R_{C}(\boldsymbol{x}, t)=\boldsymbol{\nabla} \cdot \widetilde{\boldsymbol{u}}
\end{aligned}
$$



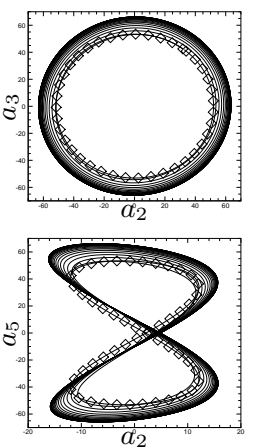

(a) $N_{r}=5$
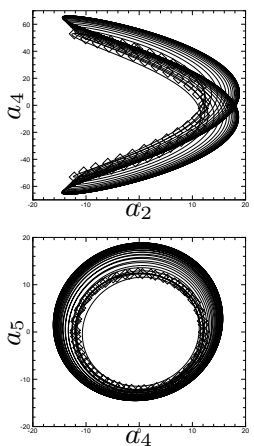

$a_{4}$

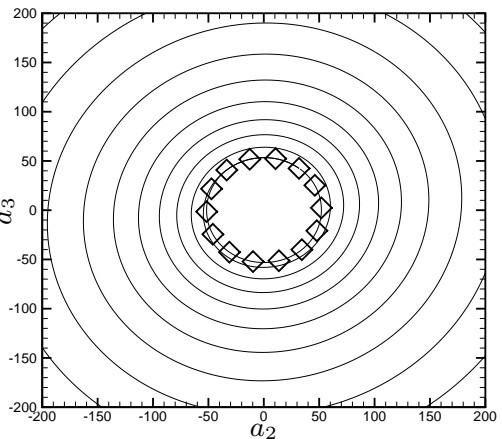

(b) $N_{r}=3$

Fig. 2. Comparison of the NS $\diamond$ and the ROM — limit cycles.

We look for the missing scales $\boldsymbol{u}^{\prime}(\boldsymbol{x}, t)=\boldsymbol{u}(\boldsymbol{x}, t)-\widetilde{\boldsymbol{u}}(\boldsymbol{x}, t)$ and $p^{\prime}(\boldsymbol{x}, t)=$ $p(\boldsymbol{x}, t)-\widetilde{p}(\boldsymbol{x}, t)$, where $\boldsymbol{u}$ and $p$ denote the exact fields. Since the resolution of the fine scales equations requires high computational costs, the objective is to derive stabilization methods based on approximations of these fine scales.

\subsection{Residuals based stabilization method: model $B^{\left[N_{r} ; K\right]}$}

The goal of this method is to approximate the fine scales $u^{\prime}$ and $p^{\prime}$ onto some adapted basis functions. The method is the following.

1. Integrate the ROM $A^{\left[N_{r}\right]}$ to obtain $N_{s}$ coefficients $a_{i}\left(t_{k}\right), k=1, \ldots, N_{s}$.

2. Compute the fields $\widetilde{\boldsymbol{u}}\left(\boldsymbol{x}, t_{k}\right)$ and $\widetilde{p}\left(\boldsymbol{x}, t_{k}\right)$, and then $\boldsymbol{R}_{M}\left(\boldsymbol{x}, t_{k}\right)$ and $R_{C}\left(\boldsymbol{x}, t_{k}\right)$.

3. Compute the POD modes $\phi_{i}^{\prime}(\boldsymbol{x})$ and $\psi_{i}^{\prime}(\boldsymbol{x})$ of $\boldsymbol{R}_{M}\left(\boldsymbol{x}, t_{k}\right)$ and $R_{C}\left(\boldsymbol{x}, t_{k}\right)$.

4. Add the $K$ first residual modes $\phi_{i}^{\prime}$ and $\psi_{i}^{\prime}$ to the existing POD basis $\phi_{i}$ and $\psi_{i}$ (using Gram-Schmidt process) and build a new ROM, noted $B^{\left[N_{r} ; K\right]}$.

\subsection{SUPG and VMS methods: models $C^{\left[N_{r}\right]}$ and $D^{\left[N_{r}\right]}$}

The streamline upwind Petrov-Galerkin (SUPG) method is a simplified version of the complete variational multiscale (VMS) method [1]. The main idea of both methods is to approximate the fine scales by $\boldsymbol{u}^{\prime} \simeq-\tau_{M} \boldsymbol{R}_{M}$ and $p^{\prime} \simeq-\tau_{C} R_{C}$. The SUPG and VMS ROMs can be formally written:

$$
\sum_{j=1}^{N_{r}} L_{i j} \frac{\mathrm{d} a_{j}}{\mathrm{~d} t}=\sum_{j=1}^{N_{r}} B_{i j} a_{j}+\sum_{j=1}^{N_{r}} \sum_{k=1}^{N_{r}} C_{i j k} a_{j} a_{k}+F_{i}(t) .
$$

- For the SUPG reduced order model, noted $C^{\left[N_{r}\right]}$, we have:

$$
F_{i}^{S U P G}(t)=\left(\widetilde{\boldsymbol{u}} \cdot \boldsymbol{\nabla} \boldsymbol{\phi}_{i}+\nabla \psi_{i}, \tau_{M} \boldsymbol{R}_{M}(\boldsymbol{x}, t)\right)_{\Omega}+\left(\boldsymbol{\nabla} \cdot \boldsymbol{\phi}_{i}, \tau_{C} R_{C}(\boldsymbol{x}, t)\right)_{\Omega} .
$$


- For the VMS reduced order model, noted $D^{\left[N_{r}\right]}$, we have:

$$
\begin{aligned}
F_{i}^{V M S}(t)=F_{i}^{S U P G}(t) & +\left(\widetilde{\boldsymbol{u}} \cdot\left(\boldsymbol{\nabla} \boldsymbol{\phi}_{i}\right)^{T}, \tau_{M} \boldsymbol{R}_{M}(\boldsymbol{x}, t)\right)_{\Omega} \\
& -\left(\boldsymbol{\nabla} \boldsymbol{\phi}_{i}, \tau_{M} \boldsymbol{R}_{M}(\boldsymbol{x}, t) \otimes \tau_{M} \boldsymbol{R}_{M}(\boldsymbol{x}, t)\right)_{\Omega}
\end{aligned}
$$

In this study parameters $\tau_{M}$ and $\tau_{C}$ are determined using optimization.

\subsection{Results of stabilization methods}

The limit cycles, over 1000 vortex shedding periods, obtained with models $B, C$ and $D$ are represented in figure 3 for $N_{r}=5$ and $N_{r}=3$ respectively and $K=2$. These limit cycles are compared to exact ones obtained by DNS. Excellent agreements are observed, thus validating our stabilization methods.
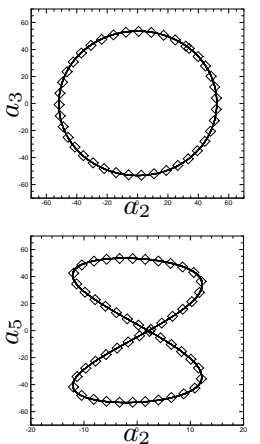

(a) $N_{r}=5$
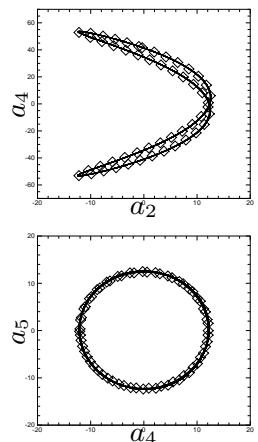

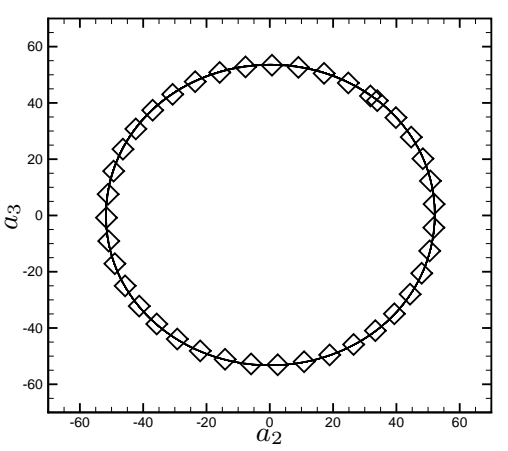

(b) $N_{r}=3$

Fig. 3. Comparison of the NS $\diamond$ and the stabilized ROM — limit cycles.

\section{Improvement of the functional subspace}

The main drawback of the POD basis is that it is only able to give an optimal representation of the snapshots set from which it was derived $[9,7,3]$. To overcome this drawback, a solution is to use a database composed by several dynamics $[3,6,4]$, but we privilege the idea of updating the POD basis during the simulation. Since each actualization requires a large computational effort (DNS), the aim of this section is to present efficient methods to actualize the functional subspace when input system parameters change. For simplicity reasons, we only focus on Reynolds number, but the forthcoming process is transposable to other input parameters. Our goal is to obtain the target basis at $R e_{2}=200$ starting from the initial basis at $R e_{1}=100$. The idea is to actualize the snapshots database replacing older snapshot with new one. A 
new snapshot is available after few DNS iterations. The corresponding POD basis, $\boldsymbol{\Phi}^{(n)}$, is computed using an efficient method (see [2]). All the ROM coefficients are built using $\boldsymbol{\Phi}^{(n)}$, and the integration is performed using $R e \equiv$ $R e_{2}$. A schematic representation of the algorithm is presented in figure 4. Figure 5 presents the evolution of the convergence criterion $\boldsymbol{\Phi}^{(n)} \cdot \boldsymbol{\Phi}^{R e_{2}}$ for

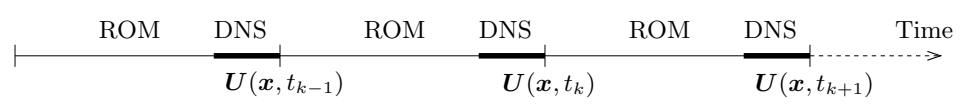

Fig. 4. Schematic representation of the hybrid DNS/ROM method.

different percentages of DNS. Denoting $T_{N S}$ and $T_{R O M}$ the time intervals where we use either DNS or ROM respectively, the percentage of DNS is $P_{N S}=T_{N S} /\left(T_{N S}+T_{R O M}\right)$. It can be seen that 10 vortex shedding periods are necessary to converge towards the target basis using only DNS $\left(P_{N S}=\right.$ $100 \%$ ). Same results can be obtained with $P_{N S}=90 \%, P_{N S}=80 \%$ and with $70 \%$ DNS. However, no convergence is obtained with $P_{N S} \leq 70 \%$. Hence, a sufficient amount of DNS is necessary to converge toward the target basis.

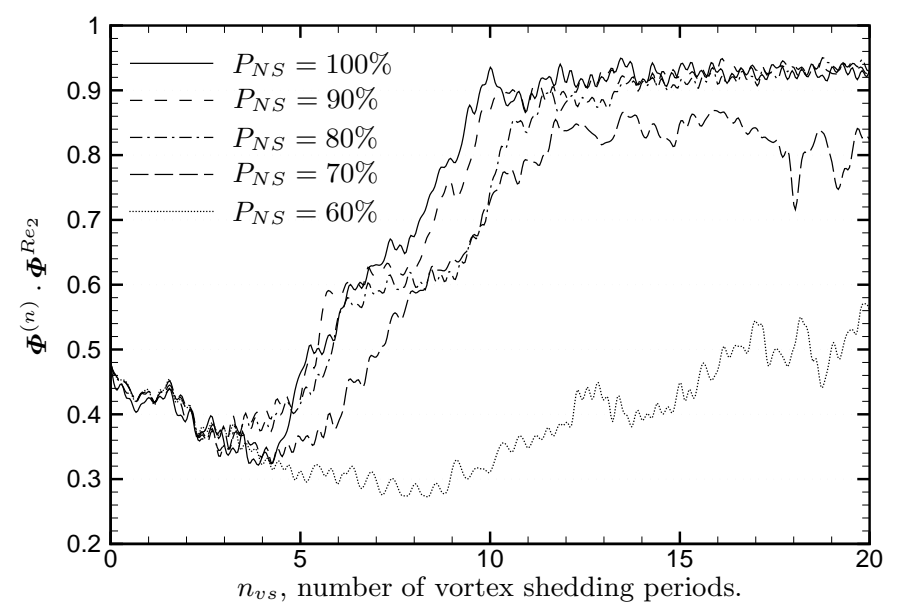

Fig. 5. Evolution of the convergence criterion for the hybrid method.

\section{Conclusions}

The objective of this paper is to improve reduced order modeling based on POD. We have built a pressure extended ROM, so that it is not necessary to model the pressure term. Although this model gives very good results, it is still necessary to model the effects of the unresolved fines scales. In this respect, we 
propose stabilization methods that consist in modeling the effect of the fine scales using residuals of Navier-Stokes operator evaluated from POD fields. The first method proposed consists in enlarging the POD subspace with few residuals modes. No empiric parameter has to be estimated in this approach. The second approach proposed relies on an approximation of the fine scale equation. Both SUPG and VMS methods give good results. In this approach, only two parameters have to be estimated. Finally, our aim is to derive an efficient method to adapt the POD basis when input parameters change. An hybrid method that couples DNS and ROMs is proposed. The idea is to update the database when dynamical evolution occurs. This method works very well if a sufficient amount of DNS is performed. Approximatively $20 \%$ of the total numerical costs can be saved using such an hybrid method.

\section{References}

1. Y. Bazilevs, V. M. Calo, J. A. Cottrell, T. J. R. Hugues, A. Reali, and G. Scovazzi. Variational multiscale residual-based turbulence modeling for large eddy simulation of incompressible flows. Comput. Methods Appl. Mech. Engrg., 197:173-201, 2007.

2. M. Bergmann, C.-H. Bruneau, and A. Iollo. Improvement of reduced order modeling based on proper orthogonal decomposition. Research Report 6561, INRIA, 062008.

3. M. Bergmann and L. Cordier. Optimal control of the cylinder wake in the laminar regime by trust-region methods and pod reduced-order models. J. Comp. Phys., 227/16:7813-7840, 2008.

4. J. Burkardt, M. D. Gunzburger, and H.-C. Lee. Centroidal Voronoi TessellationBased Reduced-Order Modeling of Complex Systems. Technical report, Florida State University, 2004.

5. B. Galletti, C.-H. Bruneau, L. Zannetti, and A. Iollo. Low-order modelling of laminar flow regimes past a confined square cylinder. J. Fluid Mech., 503:161$170,2004$.

6. X. Ma and G. E. Karniadakis. A low-dimensional model for simulating threedimensional cylinder flow. J. Fluid Mech., 458:181-190, 2002.

7. B. R. Noack, K. Afanasiev, M. Morzyński, G. Tadmor, and F. Thiele. A hierarchy of low-dimensional models for the transient and post-transient cylinder wake. J. Fluid Mech., 497:335-363, 2003.

8. B. R. Noack, P. Papas, and P. A. Monkewitz. The need for a pressure-term representation in empirical galerkin models of incompressible shear-flows. $J$. Fluid Mech., 523:339-365, 2005.

9. R. D. Prabhu, S. S. Collis, and Y. Chang. The influence of control on Proper Orthogonal Decomposition of wall-bounded turbulent flows. Phys. Fluids, 13(2):520-537, 2001.

10. D. Rempfer. On low-dimensional Galerkin models for fluid flow. Theor. Comput. Fluid Dyn., 14:75-88, 2000.

11. L. Sirovich. Turbulence and the dynamics of coherent structures. Quarterly of Applied Mathematics, XLV(3):561-590, 1987. 